\title{
Los imaginarios como punto de partida: una posibilidad de comprensión en jóvenes escolarizados desde la motricidad y el desarrollo humano
}

Imaginaries as a starting point: a comprehension possibility in schooled young people from the mobility and human development

\author{
Luis Guillermo Jaramillo Echeverri" \\ Déibar René Hurtado Herrera ${ }^{\cdots}$
}

\section{Resumen}

La clase de educación fisica la que nos permite comprender cómo los imaginarios sociales se encarnan en el área en tanto práctica cultural instituida y como discurso intencionado de formación de un cuerpo productivo, dócil y disciplinado. Pero al mismo tiempo nos acerca a los imaginarios desde los cuales los sujetos se vivencian como jóvenes, según las múltiples lecturas que hacen de la escuela.

Esta reflexión intenta resaltar los posibles horizontes desde los cuales podemos seguir construyendo nuestros imaginarios, prácticas y trayectorias a partir de los procesos de investigación que venimos adelantando en los diferentes grupos de investigación del departamento de educación fisica de la Universidad del Cauca.

Palabras clave: Sujeto, Proceso, Motricidad Humana, Educación Física, Subjetividad, Lenguaje.

\section{Abstract}

The class of physical education permits us to understand how the social imaginaries have been incorporated in this education area as an established cultural practice and a meaningful speech in a productive, docile, trained body formation. At the same time, this class approaches us near to the imaginaries from which the subjects experiment the knowledges gained from young experiences based on the many readings what young people do from school.

This reflection attempts to emphasize the possible horizons from which we can keep building our imaginaries, practices and courses of development, based on the processes of investigation which we are carrying out in many investigation groups in the Physical Education Department in the Universidad del Cauca.

Key words: $\quad$ Physical education, social imaginaries, processes of investigation.

Fecha de récepción: 3 de noviembre de 2004.

Fecha de aceptación: 16 de mayo de 2005.

Esta reflexión es parte de la investigación realizada en el grupo nacional Motricidad y Mundos Simbólicos, con la financiación de la vicerrectoria de investigaciones de la Universidad del Cauca, entre 2000 y 2004. En el grupo de Investigación participaron en calidad de investigadores Carlos Ignacio Zúñiga López y José Harvey Montoya Peláez, así como un grupo de doce estudiantes

(auxiliares de investigación) del Departamento de Educación Fisica de la Universidad del Cauca.

*.. MG. Profesor del Departamento de Educación Física, Recreación y Deporte, de la Universidad del Cauca.

*** MG. Profesor del Departamento de Educación Física, Recreación y Deporte, de la Universidad del Cauca. 


\section{Reflexión}

En principio, queremos resaltar la importancia del trayecto recorrido por los profesionales de la educación física y el nivel de madurez académica alcanzado como fruto de los procesos de formación a los que les hemos apostado.

Una forma de explicar los cambios que han venido sucediendo en el área se ubica en los procesos formativos de quienes aportaron a la formación de los licenciados en educación física en el país. Diríamos entonces que primero fueron deportistas, ex militares, técnicos en el deporte quienes desde una perspectiva deportivizada reproducían sus saberes funcionalistas y técnicos en los programas de licenciatura en educación física. Así, los estudios en el área (especializaciones, maestrías y doctorados, estudios cursados con frecuencia en el exterior) que realizaban nuestros colegas estaban en su mayoría ubicados en lo que se llamó ciencias del deporte (fisiología del ejercicio, entrenamiento deportivo, pedagogía del deporte). Otros profesionales, por el contrario, transitaron otros caminos de formación muy relacionados con las ciencias humanas y sociales como la educación, el currículo, la pedagogía y el desarrollo humano.

Este último sendero de formación marcó una diferencia significativa acerca de cómo se piensa actualmente la educación física y con ella sus procesos de formación, investigación, así como la responsabilidad que le asiste a la universidad y a sus actores institucionales. Así, desde múltiples escenarios de sentido empezaron a cuestionarse las lógicas instrumentales y mecánicas de cómo se estaba pensando al sujeto de la educación y en sí de la educación física; los dualismos en las concepciones de una realidad fragmentada entre razón y pasión, la mirada del cuerpo como anexo o extensión, como un instrumento, y las lógicas del deporte que se reprodujeron miméticamente en el escenario escolar.

Fruto de estas reflexiones, empieza a aparecer en el país un grupo de académicos que reflexionaron sobre la construcción social del cuerpo, las miradas holísticas de lo humano; el deporte con perspectiva crítica, el soma, las prácticas culturales y los imaginarios de vida que giraban en torno a una educación física como disciplina pedagógica. Sus búsquedas transitaban por la filosofía del cuerpo, la sociología, la pedagogía, la antropología, el currículo, el desarrollo humano, empezando así a impactar los programas de educación física y cuestionando de ella su lógica instrumental reproduccionista de contenidos yuxtapuestos acríticamente.
En este contexto, empiezan a emerger investigaciones de tipo comprensivo que desde la investigación histórica, la etnografía reflexiva, la complementariedad etnográfica y algunos trabajos de investigación acción participación (IAP), se ocupaban de la interpretación y transformación del área, colocándonos en otro lugar y en otra mirada del ser y hacer de la educación física. Trasegar por dichas comprensiones nos ha puesto en la evidencia y, a la vez, en la demanda, de que si no es con la participación activa de los actores implicados en los procesos de formación del área y de otras disciplinas, difícilmente podemos seguir acertadamente hacía una educación física que concibe al ser humano como ser en el mundo, como agente-constructor de una realidad que no les es ajena, la cual necesita de su actuación para ser cambiada.

Diremos, entonces, que nuestra propuesta se ubica, de manera intencional, en recuperar y visibilizar a los sujetos sociales que vivencian su corporeidad de múltiples formas. Así, preguntarnos por los imaginarios juveniles escolarizados de la educación física implicó desvelar (quitar el velo) las opacidades ${ }^{1}$ que circulan permanentemente en las voces, en los relatos, en los silencios y en lo inefable de las jóvenes y los jóvenes, de su discurso institucional y de las prácticas pedagógicas de los maestros, padres/madres, e incluso de los mismos investigadores.

De los estudios realizados en los últimos años ${ }^{2}$, entre ellos el dador de esta reflexión, podremos aventurarnos a decir que las opacidades del imaginario de la educación física se ubican alrededor de bucles como: cuerpomáquina y encarnamiento experiencial ${ }^{3}$-consumo.

Los jóvenes a través de las prácticas deportivas (que no sólo se circunscriben a la clase de educación física, aunque las contiene) vivencian su corporeidad en un juego de relaciones difíciles de delimitar entre la producción y el consumo; cuerpo-máquina que se prepara funcionalmente desde lógicas de eficiencia y eficacia, desde la repetición y la técnica, el proyecto a largo plazo y la obediencia; a su vez, es un cuerpo atlético y saludable que el capitalismo avanzado ha convertido en ideal estético y que alrededor de patrones culturales de belleza lo ubica en los terrenos del consumo.

La escuela, y en especial, la educación física, nutren y garantizan la producción de un cuerpo-objeto para que motrizmente pueda responder a las exigencias del mundo del mercado (idealización estética). La propuesta deportivizada de la educación física se encuentra en consonancia con dicho proyecto; su vigencia en la escuela y en los imaginarios de deseo que las jóvenes y 
los jóvenes expresan se relaciona estrechamente con las modalidades de producción que se ofrecen a través de la industria cultural y que invaden una ecología social de deporte en la representatividad, los nacionalismos, en el triunfo y en el ideal estético de cuerpo-consumo. Así, las modalidades de producción de cuerpo-venta nos hacen afirmar que aspectos como "la libertad de hacer, la inventiva y la creatividad son incentivadas y puestas permanentemente a prueba bajo el baremo de la competitividad" (Martín-Barbero, 2004: 37).

Pero, a su vez, emergen prácticas culturales desde las cuales los jóvenes y las jóvenes intentan fugarse a través de actividades asociadas al riesgo y la aventura, de expresiones estéticas transgresoras y nuevas sonoridades, entre otras. Si bien "el imaginario de cuerpo aparece como el objeto de distintas inspiraciones y manipulaciones científicas y tecnológicas, también es meta de nuevas prácticas de comunicación. Los jóvenes así acuden a múltiples incisiones corporales en formas de tatuajes o aretes en distintos lugares de su anatomía, hasta perforaciones en las lenguas y ojos" (Silva, El Tiempo; 2004). Son jóvenes que emergen desde lo imaginarlo y emocional, reaccionando frente a instituciones tradicionales (escuela, familia, Iglesia) que permanecen aferradas a los dualismos expulsando el cuerpo de su sensibilidad.

Frente a estas realidades mutantes que viven nuestros jóvenes, la escuela y, en especial, la educación física no han logrado desbordar la propuesta de formación de cuerpo-objeto ni cumple con la promesa de consumo que ofrecen frenéticamente los medios de comunicación. Esta invisibilización de las nuevas sensibilidades de nuestros jóvenes pone en evidencia el rezago no sólo de la escuela, sino de una educación física deportivizada que nació en los albores de una modernidad dual de la cual, no ha podido aún desprenderse.

Ahora bien, si los imaginarios que tienen las jóvenes y los jóvenes de la educación física se encuentran explícitamente deportivizados, es vano intentar resignificar las prácticas de la educación física, por cuanto ellas están ancladas en un paradigma eficientista de cuerpofísico. Las dificultades de la educación física para configurar una didáctica propia se relacionan estrechamente con el obstáculo epistemológico que le es inherente al configurar al ser humano escindido entre el pensar y el actuar, el ser y el hacer. Los procesos desde los cuales se han formado los profesionales y los formadores de formadores, en nuestro caso los "educadores físicos" (que educan "educadores físicos"), reproduce plenamente las características del paradigma de la simplicidad, es decir, la fragmentación, la disociación, la reducción atomi- zante, el control, la predicción; un modelo que lleva al entrenamiento de un licenciado (formador) con habilidades y destrezas que lo hacen ser un operario eficiente y eficaz, pero sin autonomía. Un modelo de formación con acción instrumental en el ejercicio de una educación física/deporte, donde se vivencian pocos espacios para la creatividad y la inventiva.

Las exigencias del mundo contemporáneo, de un mundo globalizado que ha hecho tránsito de la era de la información a la era del conocimiento, obliga a que nuestras instituciones escolares (en especial la universidad) se pregunten sobre los ajustes que deberán sufrir sus currículos para poder formar a sus estudiantes; sobre el tipo de competencias en las cuales se quiere formar, el tipo de relación que deberán establecer con ellos, y las estrategias que garantizarán su permanencia en el mundo del trabajo, que cada vez se hace menos promisorio en las nuevas configuraciones de sentidos de vida.

La complejidad de nuestra sociedad actual hace que no sólo los programas de educación física sean pensados, sino toda la institución universitaria y, en consecuencia, todos los programas de formación. Ante las mutaciones del sistema-mundo y en especial frente al primado de un sinnúmero de situaciones paradójicas, problémicas y desafiantes, nuestra sociedad se presenta como un locus donde la complejidad parece ser su rasgo distintivo, pues los fenómenos, al padecer una suerte de entrecruzamientos y afectaciones múltiples (donde lo lineal, lo simple, lo unidireccional, la especialidad, dan paso a lo complejo, multidimensional, interdisciplinario y transdisciplinario), requieren un arsenal cognitivo-emotivo más amplio, con el cual puedan aprehenderse y resolverse los diversos conceptos problemáticos de localidad-globalidad.

La complejidad para pensarnos a nosotros mismos "implica un rearme intelectual o cognitivo, atravesado por lo contextual, lo global, lo multidimensional, lo complejo, lo simbólico, etc., donde la especialización e hiperespecialización han terminado siendo impertinentes, inadecuadas e insuficientes ante los cambios del ejercicio profesional, la diversidad de los contextos de desempeño y las múltiples formas de vida posibles en los diversos modos de estar juntos" (Quijano, 2002: 52).

Se hace necesario entonces un cambio paradigmático que va desde el paradigma de la simplicidad (Sergio, 1999) o paradigma de la simplificación/exclusión (Mera, 2001) al paradigma de la complejidad. ¿Qué significa un proceso formativo desde el paradigma de la complejidad? En principio la complejidad (complexus) signifi- 
ca tejido, urdimbre, red, lo que está unido en conjunto; "el entramado de eventos, interacciones, retroacciones, acciones, azares, determinaciones/indeterminaciones, que constituyen nuestro mundo fenoménico" (Mera, 2001: 6).

La complejidad es un desafío, un enfoque, una perspectiva que establece y restablece las conexiones, las articulaciones, las integraciones, pero que a la vez le dan paso a la distinción (diferenciación), a la contradicción, a lo uno/diverso, al antagonismo/complementariedad. Desde esta perspectiva, formar en este paradigma significa educar para la incertidumbre como nos lo sugiere Morin (2001) en Los siete saberes necesarios para la educación del futuro; para un pensamiento de orden superior (Lipman, 1997) y para la práctica reflexiva (Shön: 1992, 1998), donde se encuentra presente el pensamiento crítico y creativo.

Los planteamientos anteriores y las nuevas búsquedas teórico-prácticas y epistemológicas han coincidido con la propuesta contemporánea de la Motricidad y el desarrollo humano, la cual considera al sujeto como ser en acción desde una perspectiva fenomenológica, e intenta rescatarlo de los reduccionismos cosificantes a los que había sido sometido. Para ello, hemos puesto sobre la mesa nuestros propios avances, la complementariedad etnográfica como una apuesta de enfoque de investigación en ciencias sociales (Murcia y Jaramillo, 2004) y la motrícidad como posibilidad de ser sujeto en acción.

La participación de nuestra comunidad académica en la construcción de la nueva ciencia de la motricidad es a nuestro juicio un camino y un compromiso para desarrollar una praxis transformadora que puede permitirnos apostarle a un proyecto educativo y humano. No se trata de hacer de la educación física, motricidad humana de manera ecléctica ni un cambio o transposición de térmínos, se trata más bien de desarrollar un discurso crítico y emancipador que desde la pregunta epistemológica y haciendo tránsito por Marleau-Ponty, Zubiry, Morin, Levinas, Derrida y otros, construya y deconstruya su conocimiento. La motricidad y el desarrollo humano es una ciencia que obliga a realizar una ruptura epistemológica, un corte que le permita su desarrollo de manera autónoma, como diría Manuel Sergio (1999): la motricidad humana sin la educación física no tiene historia, pero a su vez, la educación física sin motricidad humana no tiene futuro.

Pero qué es la motricidad humana sino una forma concreta de relación del ser humano consigo mismo, con el mundo y con sus semejantes, una forma de relación que se da a través de nuestra corporeidad, de acción humana caracterizada por la intencionalidad y por el significado. La motricidad se configura, parafraseando a Kolyniak (2003), como proceso, cuya constitución envuelve la construcción del movimiento intencional desde la reflexión, y la creación de nuevas formas de interacción a partir de la reproducción de patrones aprendidos de la acción contextualizada en la historia; por tanto, relacionada al pasado vivido y al futuro proyectado; expresando y construyendo la totalidad de las múltiples y complejas determinaciones de la continua construcción de lo humano.

Un corte epistemológico implica una voluntad imparable de construir el futuro, un acto transformador para pensar los referentes desde los cuales construir academia, así como las estrategias didácticas que pueden hacer posible una praxis transformadora. Es apenas natural que este proceso signifique alejamiento de los referentes desde los cuales fuimos formados e ingresemos al desarrollo de un pensamiento crítico y creativo (pensamiento de orden superior en términos de Lipman) que nos permita asumir los referentes que son consecuentes con el nuevo paradigma.

Tendrá entonces la motricidad humana que potenciar la gran oportunidad que la educación física tiene en sí misma debido a que, a pesar de todo, las niñas y los niños, las jóvenes y los jóvenes siguen viendo en ella un espacio de encuentro relacional y la oportunidad de romper con la cotidianidad monótona de las clases en su espacio escolar. La motricidad deberá construir una didáctica a partir de una propuesta que puede ser denominada como "Modelo Complejo" (Toro, 2003).

\section{Avanzando en la configuración}

A partir de la experiencia hemos aprendido que se hace necesario trabajar conjuntamente en la construcción de este nuevo paradigma valorando sobre todo a los demás, ya que, aunque cuestionemos sus actuaciones y algunos de sus enunciados, nos asiste la capacidad del diálogo, pero también la conciencia crítica para avanzar en nuestras reflexiones. De este modo, comprendemos que esta realidad no la transformamos si no somos capaces de comprometernos en salir del antiguo paradigma en el cual fuimos formados y reconocer que los linderos entre lo propio y lo no propio cada vez son más difíciles de establecer en tanto se hacen pertinentes para comprender nuestras realidades globales y locales en un ejercicio de fusión de horizontes.

Por tanto, nuestra propuesta aboga por una perspectiva que se fundamenta en la comprensión de las subje- 
tividades, en las posibilidades estéticas del sujeto y en los múltiples lenguajes, entendidos estos últimos como posibilidad de ser en el mundo. Por una motricidad en la que se recuperen los recorridos y experiencias corpóreas y las formas en que ellas permiten la configuración de ser sujeto. En las posibilidades de construcción como actores sociales y sujetos de derechos. Creemos que sólo así puede llegarse a una hermenéutica crítica, en tanto el sujeto logra transformar unos modos de ser y de actuar de cara a la construcción del sí mismo. No compartimos la idea de una motricidad adulto-céntrica hecha para las jóvenes y los jóvenes, en tanto ellas y ellos vivencian su corporeidad de múltiples formas, lejanos de los escenarios institucionales, de los ideales ingenuos de trascendencia y de una relación estrecha con el consumo.

En primer lugar, hablar de subjetividades implica reconocer la existencia de un sujeto que es proceso, movimiento y síntesis de aquello que Deleuze (2002) denominó sus principios constitutivos (la creencia y la creación). Esto conduce a reconocer un sujeto que se moviliza entre lo instituido (lo dado) y lo instituyente (lo creado), de tal manera que se hace sujeto en la medida en que se supera (trascendencia), reflexiona y se reflexiona; es decir, un sujeto que es creencia en tanto infiere cosas de la naturaleza que no le están dadas, e inventa distinguiendo poderes y construyendo totalidades, un ser abocado a su subjetividad. Ella (la subjetividad):

es siempre condensación de recorridos y memorias, de voces y aspiraciones en cierto sentido colectivas; se constituye siempre en la trama de relaciones con lenguajes y experiencias múltiples, pero sobre todo, en el entramado de otras subjetividades; acontece y se pronuncia con la carga de historias y biografias, de otras palabras y de otras reflexiones (Huergo 2004: 17).

Esta concepción de sujeto como proceso y movimiento permite reconocer que, en su reflexividad experiencial, va perdiendo su condición de sujeto-pasivo para mutarse en un sujeto-activo; lo que Touraine (2000:67) denominaría un sujeto que desea ser actor:

El sujeto no es una simple forma de la razón. (Éste) sólo existe al movilizar el cálculo y la técnica del mismo modo que la memoria y la solidaridad, y sobre todo al combatir, indignarse, esperar, inscribir su libertad personal en las batallas sociales y liberaciones culturales. El sujeto, más aún que razón, es libertad, liberación y rechazo.

El sujeto se hace de este modo en los desgarramientos de la experiencia vivida individualmente, en la vivencia de sus contradicciones, en sus tránsitos identitarios y en sus múltiples temporalidades; "la idea de sujeto no crece en los invernaderos demasiado protegidos. Es una planta silvestre" (Touraine, 2000: 67). En este sentido, la motricidad deberá reconocer que el sujeto en sus experiencias de vida se mueve entre lo instrumental y lo trascendente, en un proceso contradictorio pero no incoherente que lo hace activo y que busca subjetivación a través de procesos de individuación.

La subjetivación la entendemos como Foulcault denomina las tecnologías del yo, o sea, como aquellas prácticas:

que permiten a los individuos efectuar por cuenta propia o con ayuda de los otros cierto número de operaciones sobre su cuerpo y su alma, pensamientos, conducta o cualquier otra forma de ser, obteniendo así una transformación de sí mismos, con el fin de alcanzar cierto grado de felicidad, pureza, sabiduría o inmortalidad (Foucault, 1996: 48).

Prácticas donde el ser humano es consciente de la historicidad de todo presente, donde el conocimiento de sí, del sujeto por sí mismo, es el conocimiento de un ser experiencial cuyos itinerarios son construidos en relación con los otros sujetos en situación y con intencionalidad.

La subjetivación como producción de sí mismo no se restringe sólo a la experiencia ni a la búsqueda de trascendencia sino a un proyecto de vida personal que se convierte en el escenario donde pueden combinarse los dualismos de identidad-instrumental, lo técnico y lo simbólico, la razón y la pasión; de ahí la importancia de indagar por los imaginarios, ya que ellos nos pueden dar cuenta de los procesos de desgarramiento a partir de los cuales el sujeto apropia lo instituido y crea sentidos en lo instituyente. De este modo, a la subjetivación no le cabe otro contenido distinto que la creación del sí mismo, creación de sentidos de vida.

Así, los imaginarios instituidos de educación física, de cuerpo y deporte son los que nos han permitido avanzar hacia otro paradigma que recupera los procesos de subjetivación que no había tenido en cuenta la educación física. No se trata de ignorar los procesos a través de los cuales las nuevas tecnicidades generan nuevas sensibilidades, se trata de visibilizar un sujeto que en la multi-dimensionalidad de sus prácticas y experiencias construye y deconstruye su proyecto de vida. La motricidad no puede ignorar los procesos de subjetivación ya que puede caer en la invisibilización del sujeto como proceso, tal como lo hizo y lo ha hecho la educación física al ignorar las mutaciones que sufren los sujetos, los tránsitos y los recorridos por los cuales los seres hu- 
manos generan nuevas formas de ser en medio de unas culturas y las estéticas que también mutan y se renuevan permanentemente.

En segundo lugar, una propuesta de motricidad como un quiebre epistemlógico de la educación física implicaría la visibilización del sujeto como ser estético ${ }^{1}$, entendido éste como un camino para visibilizar la creación, ubicándola en un proceso de vida cotidiana. Hacer visible al sujeto es comprender las prácticas de su autoformación, las formas en que los seres humanos hacen de la vida propia una obra de arte.

En tal sentido es iluminador el planteamiento de Castoriadis (2003: 26) cuando en "tiempo y creación" afirma que:

\begin{abstract}
(...) un sujeto no es nada sino es la creación que él protagoniza de un mundo en clausura relativa [...] Esta creación es siempre creación de una multiplicidad. Esta multiplicidad se despliega siempre de dos modos: al modo de lo simplemente diferente, como diferencia, repetición, multiplicidad ensídica (conjuntista-identitaria); y al modo de lo otro, como alteridad, emergencia, multiplicidad creati$\mathrm{va}$, imaginaria o poética.
\end{abstract}

Se trata de la esencia creativa y sensible que tiende a emparentarse con la del proceso artístico, sin reducirlo a las bellas artes pero sin obviarlas, y que impulsa a la autocreación de mejores seres humanos. "La estética de la existencia tendría que ir más allá, ir donde las prácticas y los ejercicios de sí conduzcan a la creación de nuevos modos de existencia" (Marín y Muñoz, 2003: 52), es a esto que Manuel Sergio denomina "una praxis transformadora". Es la producción creativa de nuevas subjetividades y la búsqueda y generación de otras posibilidades en los dominios de lo ético, lo político, de los saberes convertidos en praxis y de lo artístico... el despliegue de procesos de creación en todas sus áreas, ese trabajo sobre "lo ya existente y siempre sobre lo que podría ser foco de resistencia frente a la homogénesis (universos de referencia unidimensional) a favor de los procesos de creación, de lo único e irrepetible (procesos de singularización)" (Marín y Muñoz, 2003: 61) un conocimiento construido a partir de la experiencia vital.

\section{Bibliografía}

BAUMAN, Z. (2003). Trabajo, consumismo y nuevos pobres. Barcelona. Gedisa.

CABREJO, P. E. (2004). La cadena simbólica de la lengua: hallazgo del eslabón perdido. En Revista Internacional Magisterio. Educación y Pedagogía. Bogotá. Cooperativa Editorial Magisterio. No. $10,7-11$.
En tercer lugar, nos quedan los lenguajes como esa posibilidad de fantasía/concrecional que nos permite apropiarnos de un mundo del cual somos y hacemos parte. La motricidad debe rescatar los diferentes modos lenguajeados que nos ubica en el aquí y en el ahora como seres configurados entre sí a través de múltiples manifestaciones. El lenguaje, al ser dinámico y activo, genera conciencia de sí y permitir contrastar continuamente manifestación y significación de las cosas. "A través del lenguaje, se empieza a utilizar una forma de tiempo socialmente organizada: se escapa de la naturaleza, creando al interior del tiempo físico, el tiempo de la cultura... el lenguaje es un enigma. Pero el enigma del lenguaje es el enigma del hombre" (Cabrejo, 2004: 8), nuestro enigma.

Por tanto, en la infinitud del lenguaje, la motricidad bucea en medio de los imaginarios de los sujetos con el propósito de entender lo que nos quieren decir desde sus estados de conciencia (fenomenología), comprender la maraña de sus significados y hacer copresencia en sus procesos de transformación. No se trata de interpretar para comprender cómo viven o vivimos cotidianamente; se trata de comprender para transformar; Heidegger lo llamaría para derribar formas de apropiación que se sedimentan en nuestras posibilidades de ser y de actuar. Porque no existe habla sino que hablamos, es que podemos desde la praxis ser proceso de cambio y no agentes externos que formulan políticas de cambio sin el compromiso activo del otro que hemos intentado comprender. Es a partir de la fusión de horizontes dialógicos que podemos ser sujetos no domesticados, "salvajes" que se resisten a ser investigador o investigado, sujeto u objeto de investigación, constructor o construido; sino que por medio del lenguaje se viven a intensidad nuestras diferentes formas de expresión. Entendemos el lenguaje desde la motricidad humana como "la comunicación de las almas-espíritu corporalizado, cuerpo espiritualizado-en el intercambio de los sentidos dados al mundo; es el sentido encarnado; es la encarnación de la experiencia en el mundo. Todo ello se proyecta, se vuelve proceso, desenvolvimiento; y al cabo, significado" (Vargas G, 2003: 14).

CASTORIADIS. C. C. (2003). “Tiempo y creación”. En Revista Anthropos. Huellas del conocimiento. Barcelona. Aula Tercer Milenio. No. 198, 25-44.

CERTEAU, M. de. (2000). La invención de lo cotidiano I. Artes de hacer. Universidad Iberoamericana. México. Iberoamericana. 
DELEUZE, G. (2002). Empirismo y subjetividad. Barcelona. Gedisa. FOUCAULT, M. (1996). Tecnologias del yo y otros textos afines. Barcelona. Paidós.

HUERGO. J. (2004). La formación de sujetos y los sentidos político-culturales de comunicación/educación. En Debates sobre el sujeto. Perspectivas contemporáneas. Fundación Uni-versidad Central, Departamento de Investigaciones DIUC. Bogotá. Siglo del Hombre Editores.

JARAMILLO, MURCIA y PORTELA. (2005). La educación física: ¿un problema de preparación o seducción? Un estudio comprensivo en el departamento de Caldas. Armenia. Kinesis.

KOLYNIAK CAROL. (2003). Proposta para um glossário inicial para a ciência da Motricidade Humana. En Revista Digital de Motricidad y Desarrollo Humano Con sentido. Número 1. Departamento de Educación Física y Recreación. Universidad del Cauca. Popayán: www.consentido.unicauca.edu.co.

LARROSA, J. (1998). La experiencia de la lectura. Estudios sobre literatura y formación. Barcelona. Leartes.

LIPMAN, M. (1997). Pensamiento complejo y educación. Madrid. Ediciones de la Torre.

MARÍN M. y MUÑOZ G. (2003). Secretos de mutantes. Música y creación de las culturas juveniles. Fundación Universidad Central. Bogotá. Siglo del Hombre Editores.

MARTÍN-BARBERO, J. (2004). Crisis identitarias y transformaciones de la subjetividad. En Debates sobre el sujeto. Perspectivas contemporáneas. Fundación Universidad Central, Departamento de Investigaciones DIUC. Bogotá. Siglo del Hombre Editores.

MERA, H. (2001). Las competencias en el ámbito educativo y de la formación profesional. Una propuesta desde la perspectiva del pensamiento complejo. Documento de trabajo. Cali.

MORIN, EDGAR. (2001). Los siete saberes necesarios para la educación del futuro. Bogotá. Cooperativa Editorial Magisterio.

MURCIA, N., JARAMILLO L. G, CAMACHO H. y LOAIZA, M. (2005) Imaginarios de los jóvenes ante la clase de educación física. Informe Final. Colciencias. Armenia. Kinesis.
MURCIA, N. y JARAMILLO, L. G. (2004). La complementariedad etnográfica. Una guía para abordar estudios sociales. Armenia. Kinesis.

QUIJANO, O. (2002). De sueño a pesadilla colectiva. Elementos para una crítica politico-cultural del desarrollo. Popayán. Universidad del Cauca.

SERGIO, M. (2004). El deporte y la motricidad humana: teoría y práctica. Simposio Internacional de Cuerpo, Motricidad y Desarrollo Humano. Mayo 19-22. Universidad de Antioquia. Medellín.

(1999). Um corte epistemológico. Da educacao fisica a motricidade humana. Lisboa. Instituto Piaget.

SERGIO, M., COEGO, J. M., TRIGO, E., TORO, S., FERNÁNDEZ, M. (2003). Aproximaciones al concepto de motricidad humana. En Revista de Ciencias del Deporte, Educación Física y Recreación Kinesis. Armenia. Kinesis. No. 36, 22-27.

SHÖN, D. (1992). La formación de profesionales reflexivos. Hacia un nuevo diseño en la enseñanza y en las profesiones. Madrid. Paidós.

(1998). El profesional reflexivo. Cómo piensan los profesionales cuando actúan. Buenos Aires. Paidós.

SILVA. A. (2004). Arte e imaginarios globales. Lecturas de fin de semana (noviembre 6) Bogotá. El Tiempo.

TOLEDO, N. U. (2001). Ejercicio de construcción de un ideal-tipo de la vida social. El caso del emprendedor. versión electrónica En Revista Cinta de Moebio No. 12. Chile. http://rehue.csociales.uchile. $\mathrm{cl} /$ publicaciones/moebio/12/frames $05 . \mathrm{html}$

TORO, A. S. (2003). Psicología de la acción. En Revista digital de motricidad y desarrollo humano consentido. Númerol. Universidad del Cauca. www.consentido.unicauca.edu.co.

TOURINE, A. (2000). ¿Podremos vivir juntos? El destino del hombre - en la aldea global. Buenos Aires. Fondo de Cultura Económica.

VARGAS GUILLÉN, G. (2003). Fenomenología del ser y el lenguaje. Serie filosofía. Bogotá. Alejandría Libros.
1 La opacidad la entendemos a partir de la propuesta de Juan Luis Pintos, en la cual las realidades sociales se presentan en un juego de relevancias y opacidades. La opacidad se entiende como aquel lado no marcado de la distinción, aquello que está oculto y que es necesario desvelar (Pintos, 2003: 168).

2 Estudios como: el Informe nacional de los adolescentes escolarizados ante la clase de educación física, investigación realizada por profesores y estudiantes de siete universidades del pais, auspiciada por las vicerrectorias y centros de investigación de cada universidad y Colciencias (Murcia, Jaramillo, Camacho y Loaiza, 2005); El sentido de las prácticas de la educación fisica en el departamento de Caldas (Jaramillo, Murcia y Portela, 2005); La cultura corporal adolescente (Bonilla, 2000) y El cuerpo en boca de los adolescentes (Arboleda y otros, 2003).

3 El encarnamiento experiencial lo entendemos como "ese saber que no puede separarse del individuo concreto que es quien encarna. Asi, el saber de la experiencia no está, como el conocimiento cientifico, fuera de nosotros, sino que sólo tiene sentido en el modo como configura un personalidad, un carácter, una sensibilidad, o en definitiva una forma humana singular que es a la vez una ética y una estética" (Larrosa, 1998:62).

La estética del consumo gobierna hoy alli donde antes lo hacia la ética del trabajo. Para quienes completaron con éxito el entrenamiento para el consumo, el mundo es un inmenso matiz de posibilidades, de sensaciones cada vez más intensas y más profundas, en el sentido de la noción alemana de Erlebnis (vivencia), diferente de Erfahrung (Experiencia). Ambos términos, aunque con matices distintos, pueden traducirse como "experiencia": Erlebnis son "las cosas por las que atravieso a lo largo de la vida", mientras que Erfahrung es la "experiencia que me enseña a vivir". El mundo y todos sus matices pueden ser juzgados por las sensaciones y Erlebnisse que provocan; por su capacidad de despertar deseos es más justamente la etapa más placentera en el proceso de consumo, más aun que la satisfacción misma del deseo. La diferente intensidad que presenta aquel despertar de los deseos determina la forma en que los objetos, acontecimientos y personas quedan señalados en el mapa de la vida; la brújula más usada par moverse en él es siempre la estética, no cognoscitiva ni moral (Bauman, 2003: 57). 